\title{
Video-assisted thoracic surgery (VATS) for resection of metastatic adenocarcinoma as an acceptable alternative
}

\author{
Marilee Carballo • Mary S. Maish • \\ Dawn E. Jaroszewski · Amy Yetasook • \\ Karl Bauer · Robert B. Cameron · E. Carmack Holmes
}

Received: 19 March 2008/Accepted: 17 October 2008/Published online: 31 December 2008

(c) The Author(s) 2008. This article is published with open access at Springerlink.com

\begin{abstract}
Background Adenocarcinomas commonly metastasize to the lungs and can be resected using open thoracotomy or video-assisted thoracic surgery (VATS). This study reviews metastatic resections in primary adenocarcinoma patients, using both thoracotomy and VATS. We aim to compare long-term prognoses to test the efficacy and viability of VATS.

Methods A retrospective review of primary adenocarcinoma patients who underwent resection of pulmonary metastases from 1990 to 2006 was carried out. Information was obtained by chart review. Endpoints analyzed were disease-free interval (DFI), survival time, and recurrencefree survival (RFS).

Results In a total of 42 (16 male, 26 female; median age 58.5 years) primary adenocarcinoma patients, 21 patients
\end{abstract}

\section{Carballo}

David Geffen School of Medicine at UCLA, UCLA,

Los Angeles, USA

e-mail: cmarilee@ucla.edu

M. S. Maish · A. Yetasook · R. B. Cameron ·

E. Carmack Holmes

Department of Thoracic Surgery, UCLA, Los Angeles, USA

M. S. Maish ( $\square)$

UCLA Thoracic Surgery, Box 957313, 64-126 CHS,

Los Angeles, CA 90095-7313, USA

e-mail: mmaish@mednet.ucla.edu

D. E. Jaroszewski

Department of Cardiovascular and Thoracic Surgery,

Mayo Clinic, Phoenix, AZ, USA

K. Bauer

Crump Institute for Molecular Imaging, UCLA,

Los Angeles, USA underwent first pulmonary metastatic resection using VATS (7 male, 14 female; median age 57 years) and 21 using thoracotomy ( 9 male, 12 female; median age 59 years). Primary adenocarcinomas were mainly 27 colorectal (64\%) and 11 breast $(26 \%)$. Two VATS (10\%) and three open patients (14\%) had local recurrences of the original cancer. Median postoperative follow was 13.3 months [interquartile range (IQR) 4.5-32.8 months] for VATS and 36.9 months (IQR 19.3-48.6 months) after thoracotomy. Median DFI-1 was 22.3 months (IQR 13.5-40.6 months) for VATS patients and 35.6 months (IQR 26.7-61.3 months) for open patients. Second thoracic occurrences were noted in six VATS patients (median DFI-2 9.2 months), and in seven open patients (median DFI-2 21.5 months). Third thoracic occurrences were noted in one VATS patient (DFI-3 18.7 months) and in one thoracotomy patient (DFI-3 21.8 months). Odds ratio of recurrence showed $12.5 \%$ less chance of developing recurrence in VATS patients. Five-year RFS was 53\% in VATS and $57 \%$ in thoracotomy patients.

Conclusions VATS has become a viable alternative to open thoracotomy for resection of pulmonary metastases. In cases of primary adenocarcinoma, VATS showed no increase in number of thoracic recurrences, and comparable RFS. Short-term follow-up is encouraging; long-term follow-up will be needed to confirm these results.

Keywords VATS - Adenocarcinoma - Thoracotomy · Pulmonary metastases · Thoracoscopy · Cancer

Aggressive resection of pulmonary metastasis has become a more commonly accepted treatment modality for carefully selected patients. Since its introduction more than a decade ago, video-assisted thoracoscopic surgery (VATS) has been used in the diagnosis of pulmonary nodules and 
malignant pleural effusion, staging of mediastinal lymph nodes, and more recently resection of pulmonary metastasis $[1,2]$. Several short-term studies have been published demonstrating the evolution and utility of VATS for treating pulmonary cancer [3-6]. However, long-term outcomes of VATS resection of pulmonary metastases have not been studied as extensively [7, 8]. The use of VATS lobectomy for non-small-cell bronchogenic carcinoma has already shown the potential long-term benefits of VATS resection of early-stage cancer [9].

Surgical resection of pulmonary metastasis is always performed with curative intent. Therefore, we consider the prognostic factors that affect long-term outcome: complete resection of lung metastasis, longer disease-free interval between treatment of primary and diagnosis of pulmonary metastasis, and presence of single or limited number of metastatic lesions. Depending on the underlying primary cancer, and the selection criteria for surgery, the expected 5 -year survival rate of surgery with curative intent varies from $30 \%$ to $50 \%$ [10-12]. The minimally invasive approach and described advantages of VATS warrant further investigation of the utility of VATS over a longer period of time with careful attention to the above factors.

This study reviews and compares our pulmonary metastatic resections in patients with primary adenocarcinoma, using both VATS and open thoracotomy, in an effort to determine the efficacy and establish the oncologic validity of VATS as an alternative approach to open thoracotomy. This can help delineate the long-term prognoses and survival advantages of VATS, as well as offer an opportunity to define more absolute indications of VATS for lung cancer.

\section{Materials and methods}

\section{Eligibility criteria}

Patients eligible for this study were those who underwent resection of pulmonary metastasis with subsequent followup at University of California, Los Angeles (UCLA) between 1973 and 2006, and met the following criteria: (1) primary adenocarcinoma at nonthoracic location, and (2) pulmonary metastasis following resection of or concurrent with primary adenocarcinoma. Exclusion criteria were: (1) primary cancer other than adenocarcinoma, (2) thoracic primary cancer, and (3) thoracic resection of a nonthoracic occurrence. The selection of operative procedure varied according to surgeon's preferences, among a total of four surgeons.

\section{Study design}

In this comparative study, we retrospectively reviewed patients who had primary adenocarcinoma with metastasis to the lungs. Information regarding disease status and survival was reviewed over the time period defined for patients with primary adenocarcinoma. This was obtained by chart review, using the Patient Centric Information Management System (PCIMS) at UCLA. We determined whether patients met inclusion and exclusion criteria. Patients were grouped by operation completed at the time of first resection: video-assisted thoracoscopic surgery (VATS) or open thoracotomy. Patient demographic information collected included age, sex, race, smoking status, site of primary cancer, use of adjuvant therapy, comorbidities, history of other cancer, and pulmonary function status. Pulmonary function status was categorized as poor, fair or good.

Patients were also evaluated for having recurrence at the primary site, metastasis to nonthoracic sites, and pulmonary metastasis. Primary recurrence was defined as recurrence of primary adenocarcinoma after resection of the same cancer. Metastases to nonthoracic sites were defined as cancer at other sites, not including the primary site or a thoracic site. Thoracic occurrence was defined as cancer in the lung at any location, and recurrences were noted as either ipsilateral solitary, ipsilateral multiple, or bilateral multiple. Regarding thoracic resections, the features evaluated included: location of occurrence, surgical approach, type of lung resection, and use of adjuvant therapy. Patients with a second and/or third occurrence were evaluated in the same manner.

Locations of thoracic occurrence were noted as right, left or bilateral. When recurrence was detected, recurrence was treated with VATS, thoracotomy or nonsurgically. We also compare the type of surgical approach used in recurrences with the approach used in previous resections. Lastly, causes of death were considered as thoracic or nonthoracic.

\section{Baseline and treatment assessments}

Patient baseline demographic information was evaluated for comparability to address the potential for selection bias. The primary outcome was survival time, defined as date of first pulmonary occurrence to latest follow-up, or death due to cancer or other cause. The secondary outcome was recurrence-free survival (RFS), defined as date of first resection to date of second occurrence, death or latest follow-up with no occurrence between these intervals. The time intervals noted were: (1) DFI-1, disease-free interval between diagnosis of primary adenocarcinoma and diagnosis of first pulmonary metastasis; (2) DFI-2, time interval between first and second occurrences; (3) DFI-3, time interval between second and third occurrences; (4) total disease interval (TDI), the start time for which was original diagnosis of primary cancer, and the terminal event was 
latest follow-up, or date of death; and (5) postoperative follow-up, defined as date of first resection to latest followup.

\section{Statistical analysis}

All data are reported as median or frequencies, with associated interquartile ranges (IQR), 95\% confidence intervals (CI), and $p$-values calculated. Categorical data, both nominal and ordinal, were compared between groups using Fisher's $\chi^{2}$ (exact) test. Continuous data were compared for differences using Mann-Whitney $U$-test. Survival analysis and recurrence-free survival were calculated according to Kaplan-Meier method. Two-tailed $p$-values with $p<0.05$ were considered statistically significant. All statistical analyses were performed using MedCalc.

\section{Results}

Patient characteristics

Between 1973 and 2006, there were a total of 182 patients who underwent resection of lung metastasis at our institution. Forty-two of the 182 patients fitted the eligibility criteria and had at least one resection of a pulmonary metastasis during the time period between 1990 and 2006. Of these 42 patients, first pulmonary resection was performed by VATS in 21 patients and by open thoracotomy 21 patients. Resection of subsequent pulmonary occurrence was either the same or a different procedure than the first one: VATS or thoracotomy or nonsurgical.

Patients were comparable in terms of most baseline patient characteristics. Demographics of the 42 patients with primary nonthoracic adenocarcinoma are listed in Table 1. Tests for homogeneity did not show statistically significant differences between the two groups. Median age was 58.5 years for all patients. The majority of participants were female and Caucasian in both groups. There were more nonsmokers than smokers $(p=0.76)$. Post hoc analysis of sample size $(\alpha=0.05$, power 0.8$)$ was 42 calculated by survival from first thoracic occurrence, and 34 calculated by RFS.

Site of primary adenocarcinoma was primarily colorectal and breast in both groups $(p=0.66)$. The other few were hepatic $(n=1)$, ovarian $(n=1)$, pancreatic $(n=1)$, and cervical $(n=1)$. Some patients also had nonthoracic metastasis $(p=0.21)$. Few patients had local recurrence of original cancer, with only two in the VATS group and three in the open group $(p=0.63)$. Metastatic lesions were more commonly located on the right side $(p=0.51)$. Of the first thoracic occurrence, unilateral solitary, unilateral multiple, and bilateral multiple lesions occurred in $43 \%(n=9)$,
Table 1 Demographics of patients at initial pulmonary metastasis

\begin{tabular}{|c|c|c|c|}
\hline & $\begin{array}{l}\text { VATS } \\
(n=21)\end{array}$ & $\begin{array}{l}\text { Open } \\
(n=21)\end{array}$ & $p$-value* \\
\hline Age $^{\mathrm{a}}$ (years) & 57 & 59 & 0.78 \\
\hline IQR & $47-68.8$ & $52.3-63.5$ & \\
\hline $95 \% \mathrm{CI}$ & $47-68.51$ & $52.49-63.34$ & \\
\hline Sex & & & 0.75 \\
\hline Female & $14(67)$ & $12(57)$ & \\
\hline Male & $7(33)$ & $9(43)$ & \\
\hline Race & & & 0.72 \\
\hline Caucasian & $17(81)$ & $15(71)$ & \\
\hline Non-Caucasian & $4(19)$ & $6(29)$ & \\
\hline Smoker & & & 0.76 \\
\hline Yes & $10(48)$ & $8(38)$ & \\
\hline No & $11(52)$ & $13(62)$ & \\
\hline History of other cancer & & & 1.0 \\
\hline Yes & $3(14)$ & $3(14)$ & \\
\hline No & $18(86)$ & $18(86)$ & \\
\hline \multicolumn{4}{|l|}{ Comorbidity } \\
\hline Single comorbidity & $12(57)$ & $8(38)$ & 0.35 \\
\hline Three comorbidities & $4(19)$ & $2(10)$ & 0.66 \\
\hline Functional status & & & 1.0 \\
\hline Poor & $1(5)$ & $0(0)$ & \\
\hline Fair & $0(0)$ & $0(0)$ & \\
\hline Good & $20(95)$ & $21(100)$ & \\
\hline Primary cancer & & & 0.49 \\
\hline Colorectal & $12(57)$ & $15(71)$ & \\
\hline Breast & $6(28)$ & $5(24)$ & \\
\hline Other & $3(14)$ & $1(5)$ & \\
\hline \multicolumn{4}{|l|}{ Metastases } \\
\hline At diagnosis of primary & $2(10)$ & $2(10)$ & 1.0 \\
\hline Nonthoracic mets ${ }^{b}$ & $6(29)$ & $11(52)$ & 0.21 \\
\hline Recurrence of primary & $2(10)$ & $3(14)$ & 1.0 \\
\hline Site first thoracic mets & & & 0.54 \\
\hline Bilateral & $5(24)$ & $3(14)$ & \\
\hline Left & $5(24)$ & $8(38)$ & \\
\hline Right & $11(52)$ & $10(48)$ & \\
\hline Deaths & $7(33)$ & $5(24)$ & 0.73 \\
\hline
\end{tabular}

* Two-tailed $p$-value; $p<0.05$ was considered statistically significant

${ }^{\text {a }}$ All data are reported as no. (\%) unless specified otherwise

b Mets, metastases

$33 \%(n=7)$, and $24 \%(n=5)$ in the VATS group, respectively, and $14 \%(n=3), 71 \%(n=15)$, and $14 \%$ $(n=3)$ in the open group, respectively. Adjuvant therapy was received in $11(52 \%)$ patients in the VATS group, and in $8(38 \%)$ patients in the thoracotomy group $(p=0.54)$.

Follow-up was complete by December 2006. Median follow-up period from primary diagnosis was 59.9 months (IQR 32.9-96.87 months), and 27.4 months (IQR 4.87-39.9 
months) from first thoracic occurrence. No patient was lost to follow-up in either group. Median TDI was shorter in the VATS group, and this difference was statistically significant $(p=0.03)$. VATS follow-up since first resection was shorter compared with follow-up in thoracotomy (13.3 versus 36.9 months), although not statistically significantly so.

Tumor response: patients with second occurrence

Second thoracic occurrences were noted in six VATS patients; three of these patients had VATS initially then had nonsurgical treatment of second occurrence, two with chemotherapy and one with no specified adjuvant treatment (median DFI-2 84 days). Patient characteristics are listed in Table 2. Another three VATS patients were initially treated with VATS then had second occurrence treated with open thoracotomy (median DFI-2 386 days). One of the latter three patients had the second VATS procedure converted to open thoracotomy the next day in order to remove a dense mass due to adhesions. No adjuvant therapy was given. A seventh patient had a second thoracic procedure performed; however, this patient was excluded from the second occurrence group because the second thoracic resection was an excision of a noncancerous mass.

A second occurrence was noted in seven open patients (median DFI-2 655 days). Four of the seven patients were subsequently treated with thoracotomy a second time (median DFI-2 585 days), one patient with chemotherapy alone (DFI-2 655), one with VATS (DFI-2 26 days), and one with radiofrequency ablation (DFI-2 708 days).

In both groups, none of the patients had recurrence of the primary cancer. All patients in the VATS group were nonsmokers. In the open thoracotomy group, five were nonsmokers and two were smokers. Recurrences in the VATS group were two (33\%) ipsilateral solitary metastasis, one (17\%) ipsilateral multiple metastasis, and three (50\%) bilateral metastases; and recurrences in the open group were five $(71 \%)$, one $(14 \%)$, and one (14\%), respectively $(p=0.47)$.

Tumor response: patients with third occurrence

A total of three patients in this study had a third resection, however only two of the three had a thoracic recurrence. The patient who had conversion of the second VATS to thoracotomy to remove a dense mass due to adhesions was excluded from the third occurrence group. The VATS patient with a third occurrence (DFI-3 569 days) did not have metastasis outside the lung. Site of first pulmonary occurrence was on the left side and was treated with VATS without adjuvant therapy. The second occurrence, located
Table 2 Demographics of patients with second occurrence

\begin{tabular}{|c|c|c|c|}
\hline & $\operatorname{VATS}(n=6)$ & Open $(n=7)$ & $p$-value* \\
\hline $\operatorname{Age}^{a}$ (years) & 52 & 55 & 0.37 \\
\hline IQR & $40-57$ & $51-60.3$ & \\
\hline $95 \% \mathrm{CI}$ & - & $46.01-63$ & \\
\hline Sex & & & 1.0 \\
\hline Female & $5(83)$ & $5(71)$ & \\
\hline Male & $1(17)$ & $2(29)$ & \\
\hline Race & & & 1.0 \\
\hline Caucasian & $4(67)$ & $4(57)$ & \\
\hline Non-Caucasian & $2(33)$ & $3(43)$ & \\
\hline Smoker & & & 0.46 \\
\hline Yes & $0(0)$ & $2(29)$ & \\
\hline No & $6(100)$ & $5(71)$ & \\
\hline History of other cancer & & & 0.46 \\
\hline Yes & $0(0)$ & $2(29)$ & \\
\hline No & $6(100)$ & $5(71)$ & \\
\hline \multicolumn{4}{|l|}{ Comorbidity } \\
\hline Single comorbidity & $1(17)$ & $2(29)$ & 1.0 \\
\hline Three comorbidities & $0(0)$ & $1(14)$ & 1.0 \\
\hline Functional status & & & 1.0 \\
\hline Poor & $0(0)$ & $0(0)$ & \\
\hline Fair & $0(0)$ & $0(0)$ & \\
\hline Good & $6(100)$ & $7(100)$ & \\
\hline Primary cancer & & & 0.33 \\
\hline Breast & $3(50)$ & $1(14)$ & \\
\hline Colorectal & $2(33)$ & $5(71)$ & \\
\hline Other & $1(17)$ & $1(14)$ & \\
\hline \multicolumn{4}{|l|}{ Metastases } \\
\hline At diagnosis of primary & $1(17)$ & $1(14)$ & 1.0 \\
\hline Nonthoracic mets ${ }^{\mathrm{b}}$ & $2(33)$ & $6(86)$ & 0.10 \\
\hline Recurrence of primary & $0(0)$ & $1(14)$ & 1.0 \\
\hline Site first thoracic mets & & & 0.72 \\
\hline Bilateral & $2(33)$ & $3(42)$ & \\
\hline Left & $3(50)$ & $2(29)$ & \\
\hline Right & $1(17)$ & $2(29)$ & \\
\hline Site second thoracic mets & & & 0.33 \\
\hline Bilateral & $3(50)$ & $1(14)$ & \\
\hline Left & $1(17)$ & $1(14)$ & \\
\hline Right & $2(33)$ & $5(71)$ & \\
\hline Deaths & $2(33)$ & $2(29)$ & 1.0 \\
\hline
\end{tabular}

* Two-tailed $p$-value; $p<0.05$ was considered statistically significant

a All data are reported as no. (\%) unless specified otherwise

b Mets, metastases

on the right, was treated with thoracotomy without adjuvant therapy, and the third occurrence, located on the left, was treated with VATS without adjuvant therapy. This patient was still alive at latest follow-up, 40 months since first thoracic occurrence. 
In the open thoracotomy group, one patient had a third occurrence (DFI-3 663 days). The first surgery, involving both sides, was a median sternotomy with three wedge resections and no adjuvant therapy. The second occurrence, located on the right, was treated with thoracotomy with adjuvant radiation therapy. The third occurrence, located on the left side, was treated with thoracotomy without adjuvant therapy. This patient also had metastasis outside the lung, in the pancreas and spleen after treatment of his primary cancer. This patient had past history of renal cell cancer. This patient is still alive, 62.3 months since first thoracic occurrence.

The primary tumor was colorectal in both patients. Both were nonsmokers, with no comorbidities, and no recurrence of primary tumor. Second and third occurrences were at distant sites from the first occurrence.

Time to events

Median DFI-1 was 22.3 months for VATS patients, shorter than for open patients ( $p=0.07)$. Median DFI-2 and DFI-3 were also shorter for the VATS group, although a statistical difference was not found (Table 3).

\section{Overall survival}

There were seven deaths in the VATS group, three nonthoracic and four of unknown cause; and five deaths in the open group, two nonthoracic and three of unknown cause. The 1-, 3-, and 5-year survival rates after first pulmonary resection were $70 \%, 54 \%$, and $54 \%$ for VATS, respectively, and $100 \%, 94 \%$, and $78 \%$ for thoracotomy, respectively $(p=0.14$, Fig. 1$)$. Corresponding median overall survival was 13.3 months in the VATS group, and 36.9 months in the open group $(p=0.10)$. After 6 years since initial metastasectomy, survival dropped to $26 \%$ in the thoracotomy group, but remained at $54 \%$ for the VATS group. Among the patients with a single pulmonary

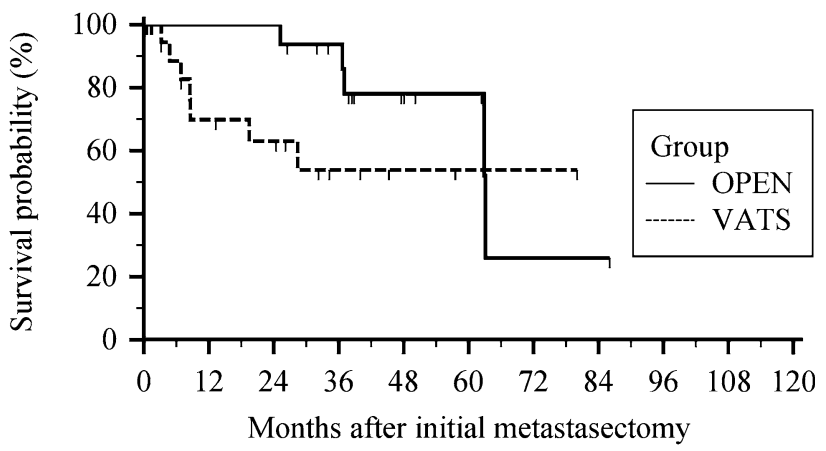

Number at risk

Group: OPEN

$\begin{array}{ccccccccccc}21 & 16 & 16 & 12 & 6 & 4 & 1 & 1 & 1 & 1 & 1 \\ \text { Group: VATS } & & & & & & & & & \\ 21 & 11 & 9 & 4 & 2 & 1 & 1 & 0 & 0 & 0 & 0\end{array}$

Fig. 1 Overall survival after initial metastasectomy, all patients

metastasis and resection, most deaths occurred within the first year in the VATS group, with 1-year survival of $64 \%$ and 5-year survival of 55\%. The 1- and 5-year survival rates in the open thoracotomy group were $100 \%$ and $77 \%$, respectively, although survival dropped to $39 \%$ after 6 years in this group $(p=0.23)$.

\section{Recurrence-free survival}

A total of six patients in the VATS group had more than one thoracic occurrence $(n=7)$, and median RFS was 8.6 months (Table 3). In the thoracotomy group, there were 7 patients $(n=8)$, and median RFS was 25.1 months. The VATS group showed no recurrences after 32 months, and the open group showed none after 35 months. Estimated RFS rate at 5 years and beyond was $53 \%$ in the VATS group, and $57 \%$ in the open thoracotomy group ( $p=0.77$, Fig. 2). The odds ratio for recurrence shows a $12.5 \%$ less chance of developing recurrences in the VATS patients (Table 4). Two thoracotomy patients developed recurrence within the first 30 days after initial

Table 3 Time intervals

* Two-tailed $p$-value; $p<0.05$
was considered statistically
significant
${ }^{\text {a }}$ All data are reported as
months (IQR) unless specified
otherwise

\begin{tabular}{llll}
\hline & VATS & Open & $p$-value* \\
\hline $\begin{array}{l}\text { Follow-up (1990-2006) } \\
\text { Total disease interval }\end{array}$ & $50.7(23.3-69.8)$ & $80.6(47.7-121.3)$ & 0.03 \\
Postoperative follow-up & $13.3(4.5-32.8)$ & $36.9(19.3-48.6)$ & 0.10 \\
Disease-free intervals & & & \\
DFI-1 & $22.3(13.5-40.6)$ & $35.6(26.7-61.3)$ & 0.07 \\
DFI-2 & $9.2(2.7-16.3)$ & $21.5(4.6-23)$ & 0.53 \\
DFI-3 & 18.7 & 21.8 & 1.0 \\
No more recurrences after & 32 & 35 & \\
Survival & & & 0.33 \\
Recurrence-free survival & $8.6(3.1-27.7)$ & $25.1(0.8-40.2)$ & 0.10 \\
Overall survival & $13.3(4.5-32.8)$ & $36.9(19.3-48.6)$ & \\
\hline
\end{tabular}




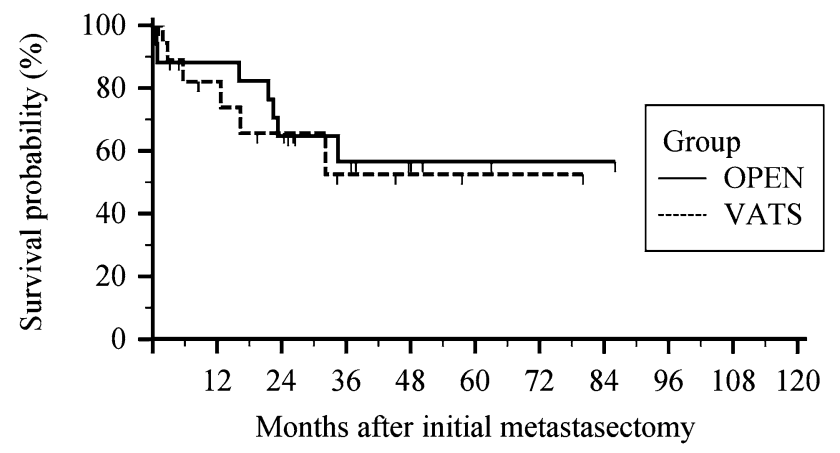

Number at risk

Group: OPEN

$\begin{array}{ccccccccccc}21 & 15 & 11 & 7 & 4 & 2 & 1 & 1 & 1 & 1 & 1 \\ \text { Group: VATS } & & & & & & & & & \\ 21 & 10 & 7 & 3 & 2 & 1 & 1 & 0 & 0 & 0 & 0\end{array}$

Fig. 2 Recurrence-free survival after initial metastasectomy, all patients

metastasectomy, while none were seen in the VATS group. Three VATS patients developed recurrence between 1 month to 1 year. Most recurrences occurred within the first 2 years in both groups (six open, five VATS). Post hoc power analysis showed 69\% power in recurrence-free survival equality analysis, and $61 \%$ power in overall survival equality analysis $(\alpha=0.05$, double sided).

\section{Discussion}

Key results

Various studies have demonstrated the benefits of VATS over open thoracotomy, making VATS a favorable modality to treat cancer: less invasive with a decreased amount of chest wall trauma, less pain, less inflammatory response and smaller degree of immunosuppression, shorter hospital stay, accelerated postoperative recovery, decreased long-term morbidity, and better long-term functional level $[2,13]$. These features, however, have been demonstrated over a short-term period, and it is not yet well documented whether the long-term advantages are comparable to standard open thoracotomy. Recognizing that complete resection remains a main determinant of survival, this study reviewed outcomes of primary nonthoracic adenocarcinoma with pulmonary metastasis treated with either VATS or open thoracotomy to initiate the evaluation of the long-term outcomes of VATS and its adequacy for cancer surgery.

This study is derived from a reasonably extended follow-up period of 59.9 months. During the period under study, 23 VATS and 29 thoracotomy procedures were done in 42 patients. Our data estimates 5-year overall survival rate from first thoracic occurrence at $54 \%$ for the VATS group and $78 \%$ in the thoracotomy group. Despite this difference, survival in the VATS patients is comparable to that in previous short-term studies. Data from the literature [10-12, 14-17] suggest that 5-year survival after resection of pulmonary metastasis with thoracotomy ranges from $32 \%$ to $50 \%$. After 6 years since initial metastasectomy, the survival rate remained at $54 \%$ in the VATS group, but dropped to $26 \%$ in the open thoracotomy group. Deaths unrelated to cancer were also included in the analysis of overall survival, which could account for the differences in survival rate. In our study, none of the deaths in the VATS group or the thoracotomy group were due to thoracic causes.

We also evaluated recurrence-free survival since we were interested in recurrence of cancer. Those patients with a second or third occurrence had subsequent resection with the same or other procedure. Our data show 5-year recurrence-free survival rate of $53 \%$ in VATS $(n=7)$, which is comparable to that in open thoracotomy $(57 \%, n=8)$. Corresponding median RFS from second and third occurrence was 8.7 and 25.5 months, respectively. In this study, the odds ratio of 0.86 showed that recurrence is $12.5 \%$ less likely in the VATS patients.

Statistical analysis demonstrated that the two groups were similar in terms of patient characteristics, enabling us to make clinically and statistically relevant comparisons. We selected patients with primary adenocarcinoma who underwent either VATS or open thoracotomy for resection of first pulmonary metastasis. From post hoc analysis ( $\alpha=0.05$, power 0.8 ), the sample size in our study is considered adequate.

Table 4 Survival after median follow-up of 59.9 months from primary diagnosis (27.4 months from initial metastasectomy)

\begin{tabular}{lcclc}
\hline & VATS $(n=21)$ & Open $(n=21)$ & $\begin{array}{l}\text { Odds ratio } \\
\text { (VATS versus open) }\end{array}$ & $\begin{array}{c}\text { 95\% CI } \\
\text { Total recurrences }\end{array}$ \\
Overall survival & $7(33)$ & $8(38)$ & 0.88 & $0.39-1.98$ \\
Recurrence-free survival & $14(67)$ & $16(76)$ & 1.4 & $0.50-3.71$ \\
\hline
\end{tabular}

* Two-tailed $p$-value; $p<0.05$ was considered statistically significant

${ }^{\text {a }}$ All data are reported as no. (\%) unless specified otherwise 
Interpretation

Surgical removal of lung metastasis is performed with curative intent, and has proven to be a therapeutic option. Virtually any tumor can metastasize to the lung, with colorectal, breast, head and neck, thyroid, and renal cell cancers among the most likely to spread to the lungs [18]. Our results parallel these finding. In this study, colorectal and breast cancer were the most common primary tumors that metastasized to the lungs, with $57 \%$ and $28 \%$ in the VATS group, and $71 \%$ and $24 \%$ in the open group, respectively. The two patients with a third occurrence had primary colorectal cancer.

The most common form of secondary lung tumors are metastatic malignant neoplasms, with the subpleural or outer third of the lung fields as the most common site, and the most typical appearance as well-circumscribed noncalcified nodules. In both groups, pulmonary metastasis was more common in the right lobe. One patient in the open group had a single recurrence that was subsequently treated with VATS, and no further recurrence was noted in this patient. Whether this is a manifestation of the type of surgery performed or the inherent behavior of the primary cancer is not clearly delineated. However, given the favorable features of VATS and that metastasis to the lung frequently presents as a solitary pulmonary nodule occurring at a single site, this study supports that pulmonary metastasis can still be considered very amenable to resection using VATS. Furthermore, with use of spiral computed tomography (CT) scan to localize metastases for surgical planning, lesions can be accurately identified, in contrast to with older CT scans that missed lesions, allowing VATS to be successful [19, 20].

A major concern regarding the use of VATS with malignancy would be potential for increased incidence of local or distant recurrence. We considered local recurrence as recurrence at the same site of prior resection. In addition, we considered recurrence in the ipsilateral lung a distant recurrence. Recurrence at a remote site in the remaining ipsilateral lung would not be related to surgical technique given it had been resected in the prior procedure. Therefore, recurrence in the ipsilateral lung was analyzed as distant recurrence. Our findings show that recurrence at the same site was infrequent, being seen in two VATS patients and in one thoracotomy patient; the remainder were distant in both groups. Both groups showed a similar rate of recurrence, although recurrence is $12.5 \%$ less likely in VATS patients. Several factors and careful selection of a surgical candidate remain an influence on the long-term outcome: ability to control primary tumor size, absence of other sites of extrapulmonary metastasis, complete resectability, adequate cardiopulmonary reserve, and technical feasibility
[18]. The implication remains that patients with multiple lesions should be treated with open thoracotomy, which does allow palpation of lung tissue and a better overview.

In addition to location of recurrence, we felt that DFI-1 would give an impression of the accuracy of VATS. Median DFI-1 was 22 months in the VATS group and 36 months in the open group. Previous studies have shown a disease-free interval ranging from 31.5 to 37.5 months [14-16, 21]. The data do not suggest a statistical difference in DFI-1 $(p=0.07)$ or DFI-2 $(p=0.53)$. Given the likelihood of recurrence in both groups, we believe that adjunctive therapies such as chemotherapy and radiation are also essential to maximize survival. Only 4 of 11 VATS patients and only 3 of 8 open patients treated with chemotherapy and/or radiation had recurrence.

\section{Limitations}

Despite our classification process, we readily admit that our method has the potential for selection bias. Although there may be bias to surgeon, each of the four surgeons has a long history of surgical experience, which almost eliminates any bias related to surgical skill. Another limitation was the small sample of patients used in this study. One recommendation for future studies is to increase the sample size, which will also increase the power of the study. Our study, similar to previous studies, is a retrospective analysis of highly selected patients. Potential limitations include the need to combine patients with nonthoracic metastasis and those without nonthoracic metastases, and inability to adequately know whether the primary tumor was controlled. For a more definitive evaluation of the advantages of VATS over open thoracotomy in a long-term setting, a larger-scale prospective randomized study testing for equivalence or noninferiority to standard therapy would be beneficial.

\section{Future and conclusion}

VATS has become a viable alternative to open thoracotomy for resection of pulmonary metastases. In the cases of primary adenocarcinoma, VATS shows a reasonable disease-free interval and shows no additional increase in the number of thoracic recurrences compared with open thoracotomy. Our retrospective study demonstrates that VATS appears to have better outcome in terms of recurrence rate, with 5-year recurrence-free survival and median overall survival comparable to open thoracotomy. Short-term follow-up is encouraging; long-term follow-up will be needed to confirm these results and to assist in establishing a final conclusion. 
Open Access This article is distributed under the terms of the Creative Commons Attribution Noncommercial License which permits any noncommercial use, distribution, and reproduction in any medium, provided the original author(s) and source are credited.

\section{References}

1. Lin J, Iannettoni MD (2003) The role of thoracoscopy in the management of lung cancer. Surg Oncol 12:195-200

2. Luh S, Liu H (2006) Video-assisted thoracic surgery-the past, present status and the future. J Zhejiang Univ Sci B 7:118-128

3. Kirby TJ, Mack MJ, Landrenaeau RJ, Rice TW (1993) Initial experience with video-assisted thoracoscopic lobectomy. Ann Thorac Surg 56:1248-1252

4. Nakata M, Sawada S, Yamashita M, Saeki H, Kurita A, Takashima S, Tanemoto K (2004) Surgical treatments for multiple primary adenocarcinoma of the lung. Ann Thorac Surg 78:11941199

5. Shiraishi T, Shirakusa T, Iwasaki A, Hiratsuka M, Yamamoto S, Kawahara K (2004) Video-assisted thoracoscopic surgery (VATS) segmentectomy for small peripheral lung cancer tumors. Surg Endosc 18:1657-1662

6. Walker WS, Carnochan FM, Pugh GC (1993) Thoracoscopic pulmonary lobectomy. Early operative experience and preliminary clinical results. J Thorac Cardiovasc Surg 106:11111117

7. Shigemura N, Akashi A, Funaki S, Nakagiri T, Inoue M, Sawabata N, Shiono H, Minami M, Takeuchi Y, Okumura M, Sawa Y (2006) Long-term outcomes after a variety of video-assisted thoracoscopic lobectomy approaches for clinical stage IA lung cancer: a multi-institutional study. J Thorac Cardiovasc Surg 132:507-512

8. Sugi K, Kaneda Y, Esato K (2000) Video-assisted thoracoscopic lobectomy achieves a satisfactory long-term prognosis in patients with clinical stage IA lung cancer. World J Surg 24:27-31

9. Walker WS, Codispoti M, Soon SY, Stamenkovic S, Carnochan F, Pugh G (2003) Long-term outcomes following VATS lobectomy for non-small cell bronchogenic carcinoma. Eur J Cardiothorac Surg 23:397-402
10. Kandioler D, Krömer E, Tüchler H, End A, Müller MR, Wolner E, Eckersberger (1998) Long-term results after repeated surgical removal of pulmonary metastases. Ann Thorac Surg 65:909-912

11. Mountain CF, McMurtrey MJ, Hermes KE (1984) Surgery for pulmonary metastasis: a 20-year experience. Ann Thorac Surg 38:323-330

12. Pastorino U, Buyse M, Griedel G, Ginsberg RJ, Girard P, Goldstraw P, Johnston M, McCormack P, Pass H, Putnam JB (1997) Long-term results of lung metastasectomy: prognostic analyses based on 5206 cases. J Thorac Cardiovasc Surg 113:3749

13. Koizumi K (2006) Current surgical strategies for lung cancer with a focus on open thoracotomy and video-assisted thoracic surgery. J Nippon Med Sch 73:116-121

14. Rena O, Casadio C, Viano F, Cristofori R, Ruffini E, Filosso PL, Maggi G (2002) Pulmonary resection for metastases from colorectal cancer: factors influencing prognosis Twenty-year experience. Eur J Cardiothorac Surg 21:906-912

15. Sakamoto T, Tsubota N, Iwanaga K, Yuki T, Matsuoka H, Yoshimura M (2001) Pulmonary resection for metastases from colorectal cancer. Chest 119:1069-1072

16. Zink S, Kayser G, Gabius HJ, Kayser K (2001) Survival, diseasefree interval, and associated tumor features in patients with colon/ rectal carcinomas and their resected intra-pulmonary metastases. Eur J Cardiothorac Surg 19:908-913

17. Casali C, Stefani A, Storelli E, Morandi U (2006) Prognostic factors and survival after resection of lung metastases from epithelial tumours. Interact CardioVasc Thorac Surg 5:317-321

18. Kondo H, Okumura T, Ohde Y, Nakagawa K (2005) Surgical treatment for metastatic malignancies. Pulmonary metastasis: indications and outcomes. Int J Clin Oncol 10:81-85

19. Chen W, Chen L, Quiang G, Chen Z, Jing J, Xiong S (2007) Using an image-guided navigation system for localization of small pulmonary nodules before thoracoscopic surgery. Surg Endosc 21:1883-1886

20. Sutedja G (2003) New techniques for early detection of lung cancer. Eur Respir J 21(Suppl. 39):57s-66s

21. Mansel JK, Zinsmeister AR, Pairolero PC, Jett JR (1986) Pulmonary resection of metastatic colorectal adenocarcinoma: a ten year experience. Chest 89:109-112 